\title{
El pramipexole vs. la levodopa tuvo menos complicaciones motoras y menos efectividad como tratamiento inicial del Parkinson
}

Pramipexole vs levodopa as initial treatment for Parkinson's disease. A randomized controlled trial. Parkinson Study Group. JAMA. 2000 oct. 18; 284:1931-38

\section{Objetivo}

Comparar el desarrollo de complicaciones motoras de la Enfermedad de Parkinson (EP) iniciando el tratamiento en el estadio temprano con pramipexole vs. levodopa.

Diseño

Ensayo clínico aleatorizado, doble-ciego, multicéntrico.

Lugar

Veintidós centros universitarios dedicados a movimientos anormales en EE.UU. y Canadá.

\section{Pacientes}

Se incluyeron 301 pacientes (edad media 61.5 años ) con enfermedad de Parkinson en su estadio temprano (sin demencia), que requirieran terapéutica dopaminérgica para tratar su incapacidad progresiva. Fueron enrolados entre Octubre de 1996 y Agosto de 1997.

\section{Intervención}

Se aleatorizó a los pacientes para recibir $0.5 \mathrm{mg}$ de pramipexole tres veces por día $(n=150)$; o carbidopa/levodopa 25/100 mg tres veces por día $(n=150)$. Para pacientes con incapacidad residual, as dosis fueron aumentándose durante las primeras diez semanas. De la semana 11 hasta los dos años, los investigadores pudieron agregar, de ser necesario, levodopa fuera de protocolo, para tratar síntomas no controlados.

\begin{abstract}
Medición de los resultados principales
El resultado principal a medir fue el momento en que apareciera cualquiera de las siguientes tres complicaciones motoras: deterioro de fin de dosis, discinesias, o fluctuaciones "on-off". Los resultados secundarios fueron: cambios en el puntaje de la escala UPDRS (Unified Parkinson's Disease Rating Scale), implementada al inicio y durante el seguimiento.
\end{abstract}

\section{Resultados}

El tratamiento inicial con pramipexole resultó en una disminución significativa en el desarrollo de complicaciones motoras dopaminérgicas ( $28 \%$ comparado con $51 \%$ en el grupo levodopa, reducción de riesgo relativo 55\% [IC95\% 30 a 67\%], p < 0.001). La mejoría en la escala UPDRS total inicial y al mes 23.5 fue mayor en el grupo levodopa ( 9.2 vs. 4.5 puntos, $p<0.001$ ). La somnolencia fue más común en los pacientes tratados con pramipexole (32.4 vs $17.3 \%, p=0.003$ ).

\section{Conclusiones}

El tratamiento inicial del Parkinson con pramipexole produjo una menor incidencia de complicaciones motoras, con respecto a los tratados inicialmente con levodopa. A pesar del suplemento con levodopa extra, fuera de protocolo, en ambos grupos, el grupo tratado sólo con levodopa tuvo mayor mejoría en el UPDRS comparado con el grupo pramipexole.

\section{Comentario}

Los agonistas dopaminérgicos han sido usados tradicionalmente como terapia coadyuvante a la levodopa en pacientes con EP, una vez que han desarrollado complicaciones motoras ${ }^{1}$. Sólo recientemente se ha investigado su introducción temprana en el tratamiento inicial de la EP.

Este estudio, confirma los hallazgos de otros dos publicados previamente con los agonistas ropirinole y cabergolina2. En este trabajo el uso inicial del pramipexole, un agonista de nueva generación, no ergolínico, disminuyó la aparición de complicaciones motoras en un 55\% (IC95\% 30 a 67\%) en relación con el inicio de tratamiento únicamente con levodopa. A su vez confirma que los agonistas conocidos y en este caso el pramipexole, son menos efectivos que el tratamiento clásico con levodopa en el tratamiento de los síntomas motores de la EP. Esto constituye un fenómeno peculiar dentro del marco de este estudio, ya que los pacientes enrolados podían luego de la semana 11 recibir levodopa fuera de protocolo según el juicio de los médicos tratantes para manejar los síntomas no controlados inicialmente. Aún así, los pacientes en el grupo pramipexole tuvieron una peor función motora que los del grupo levodopa.

Se cree que esto probablemente se deba a algún factor adicional provisto por el pramipexole y no medido por las escalas utilizadas 3 . Un ejemplo de esto último es la el hallazgo de que esta droga tiene un efecto antidepresivo similar al de la fluoxetina, en pacientes no EP4. Otro dato a destacar es que la incidencia de los efectos adversos dopaminérgicos, alucinaciones, somnolencia y edema de miembros inferiores fueron mayores en el grupo que utilizó pramipexole. En relación con el díscutido potencial neurotóxico de la levodopa y el potencial efecto neuroprotector de los agonistas dopaminérgicos $^{5}$, no se pudo demostrar diferencias significativas a favor de ninguno de los subgrupos, en relación a la evolutividad de la EP, evaluando la fijación estriatal de transportadores dopaminergicos. En síntesis, el pramipexole es una terapia eficaz en el tratamiento inicial de la EP, aunque menos eficaz que la levodopa. Su uso, como el de otros agonistas, disminuye y retrasa la aparición de complicaciones motoras. Esto lo hace a expensas de una potencia dopaminérgica menor y a una mayor incidencia de efectos adversos. Estas características, compartidas con los otros agonistas dopaminérgicos, la convierten en una estrategia particularmente atractiva para tratar a pacientes jóvenes con EP, en los que se intenta muchas veces evitar el tratamiento inicial con levopdopa. Se debe mencionar que este estudio no considera un aspecto importante en la terapia antiparkinsoniana que es el de la costo-efectividad (la mayoría de los agonistas son muy costosos). Esperemos que nuevos trabajos consideren este punto ya que es de especial relevancia en países como los nuestros.

\section{Dr. Diego Bauso [ Servicio de Neurología. Hospital Italiano de Buenos Aires ]}

\footnotetext{
Referencias

1. Rascol O, Brooks D, Korzyn AD, et al. A five year study of the incidence of dyskinesia in patients with early Parkinson's disease who were treated with ropirinole or levodopa. N. Engl $J$ Med. 2000;342:1484-1491

2. Rinne UK, Bracco F, Chouza C, et al. Early Treatment of Parkinson's disease with cabergoline delays the onset of motor complications: results of a double-blind, levodopa controlled trial. Drugs. 1998; 55 (suppl. 1):23-32.

3. Piercey MF, Camacho-Ochoa M, Smith MW. Functional Roles for dopamine-recptor subtypes. Clin Neuropharmacol. 1995;18 (suppl):534-542.

4. Corrigan MH, Denahan AQ, Wright CE. Comparision of pramipesole, fluoxetina and placeebo in patients with mayor depression. Depress Anxiety. 2000;11:56-65.

5. Olanow CW, jenner P, Brooks D. Dopamine agonists and neuroprotection in Parkinson's disease. Ann Neurol. 1998; 44 (suppl 1):167-174.
} 